\title{
Gas Cascade Amplification in Ultra-High-Resolution Environmental Scanning Electron Microcopy
}

\author{
Milos Toth ${ }^{1, *}$ Bradley L. Thiel, ${ }^{2}$ and W. Ralph Knowles, ${ }^{1, \dagger}$ \\ ${ }^{1}$ FEI Company, 5350 NE Dawson Creek Drive, Hillsboro, OR 97124, USA \\ ${ }^{2}$ College of Nanoscale Science and Engineering, State University of New York at Albany, 251 Fuller Road, \\ Albany, NY 12203, USA
}

\begin{abstract}
We describe a feedback mechanism in the gas cascade amplification process used in magnetic immersion lens environmental scanning electron microcopy (ESEM). Feedback dominates gas gain under the conditions typically used for ultra-high-resolution ESEM and gives rise to novel dependencies of the imaging signal and noise on microscope operating parameters. It is ascribed tentatively to the generation of free electrons upon de-excitation of metastable species in the gas cascade. The results have implications for optimization of ESEM systems for applications such as critical dimension metrology and real-time imaging of nanostructure growth by gas mediated electron beam induced deposition.
\end{abstract}

Key words: low vacuum SEM, environmental SEM, gas cascade amplification, metastables, magnetic field enhancement, feedback, secondary electron imaging

\section{INTRODUCTION}

Environmental scanning electron microscopy (ESEM) employs gaseous environments to charge stabilize nonconducting materials (Baker et al., 2003; Thiel \& Toth, 2005). The combination of ESEM and magnetic immersion lens (MIL) electron columns has enabled ultra-high-resolution $(\sim 1 \mathrm{~nm}$, under optimal conditions) imaging of systems such as bulk insulators, photolithographic masks, and low density nanoporous dielectrics (aerogels) (Toth et al., 2006; Kucheyev et al., 2007). Gaseous environments also enable electron imaging of liquid and hydrated materials (Stokes et al., 1998; Stelmashenko et al., 2001; Rossi et al., 2004; Bogner et al., 2008; Noscinovsky \& Bhushan, 2008) and gas mediated, electron beam induced nanofabrication (Folch et al., 1995, 1996; Molhave et al., 2003, 2004) at sub-10 nm length scales (Toth et al., 2007).

MIL ESEM uses a magnetic field assisted gas ionization cascade to amplify the secondary electron imaging signal (Thiel et al., 2006) and generate gaseous ions that suppress charging artifacts during ESEM imaging and nanofabrication of materials that accumulate excess charge under electron irradiation (Toth et al., 2006, 2007; Kucheyev et al., 2007). The gas ionization cascade has been described previously by a model of electron transport and impact ioniza-

Received January 29, 2010; accepted March 30, 2010

*Corresponding author. E-mail: milos.toth@FEI.com

${ }^{\dagger}$ Ralph Knowles has participated in the research presented here, but passed away in an unfortunate accident prior to the writing of the manuscript. tion of the gas in the presence of the electric and magnetic fields encountered in MIL ESEM (Thiel et al., 2006). Here we show that under conditions of low gas pressure $\left(<10^{2} \mathrm{~Pa}\right)$ and high gas ionization rate, the cascade amplification efficiency is much greater than that achievable solely by primary ionization processes. The additional amplification is consistent with secondary ionization processes, such as gas ionization cascades triggered by free electrons generated during the relaxation of metastable species in the gas cascade. Evidence for secondary processes derives from superexponential dependencies of the electron imaging signal and noise on the potential difference between the sample surface and the detector. The associated signal amplification, noise statistics, and ion generation all have significant implications for high-resolution imaging, ESEM metrology, and gas mediated nanofabrication.

\section{Materials And Methods}

Experiments were performed using an FEI NanoSEM 600, a field emission gun magnetic immersion lens ESEM previously described in Toth et al. (2006) and Thiel et al. (2006). A cross-sectional schematic of the detector is shown in Figure 1. Detailed discussions of the methodology used to measure the gas cascade output current signal and noise can be found in Thiel et al. (2006) and Tileli et al. (2009). $\mathrm{H}_{2} \mathrm{O}$ and electrically grounded $\mathrm{Pt}$ were used as the imaging gas and the sample. In figure captions, $E_{b}=$ beam energy, $d_{W}=$ working distance, $I_{b}=$ beam current, $B_{m}=$ peak axial 


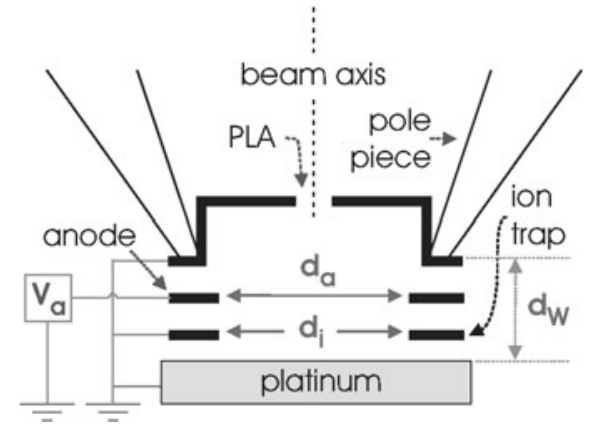

Figure 1. Schematic illustration of the detector immersed in the magnetic field of an immersion electron lens. All electrodes are symmetric about the beam axis (PLA = pressure limiting aperture, $d_{a}=$ anode diameter, $d_{i}=$ ion trap diameter, $V_{a}=$ anode bias, $d_{W}=$ working distance) .

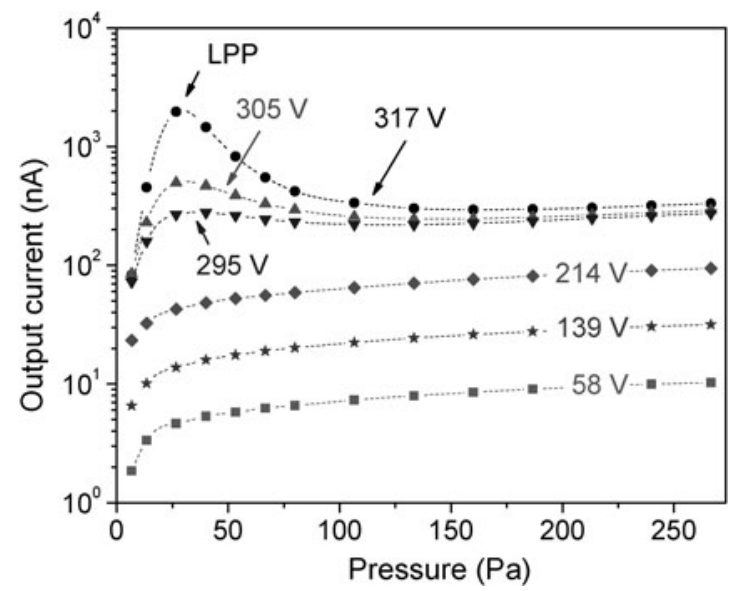

Figure 2. Gas cascade output current measured as a function of pressure at the anode biases indicated (LPP $=$ low pressure peak) $\left(d_{a}=d_{i}=2.5 \mathrm{~mm}, E_{b}=5 \mathrm{keV}, d_{W}=4 \mathrm{~mm} ; I_{b}=200 \mathrm{pA}, B_{m}=\right.$ $253 \mathrm{mT})$.

magnetic field generated by the immersion objective lens, and $V_{a}=$ detector anode bias (see Fig. 1).

\section{Results AND Discussion}

Figure 2 shows plots of the gas cascade output current measured as a function of pressure at anode biases in the range of 58 to $317 \mathrm{~V}$. At low bias $\left(V_{a} \leq 214 \mathrm{~V}\right)$, the cascade output current (which is proportional to gas gain $G$ ) increases gradually with pressure $(P)$. This behavior is qualitatively the same as when $\mathrm{N}_{2}$ is used as the amplification gas, and can be explained and modeled completely by primary gas ionization processes (Thiel et al., 2006). However, at anode biases greater than approximately $250 \mathrm{~V}$, the $G(P)$ profiles shown in Figure 2 exhibit an anomalous gain
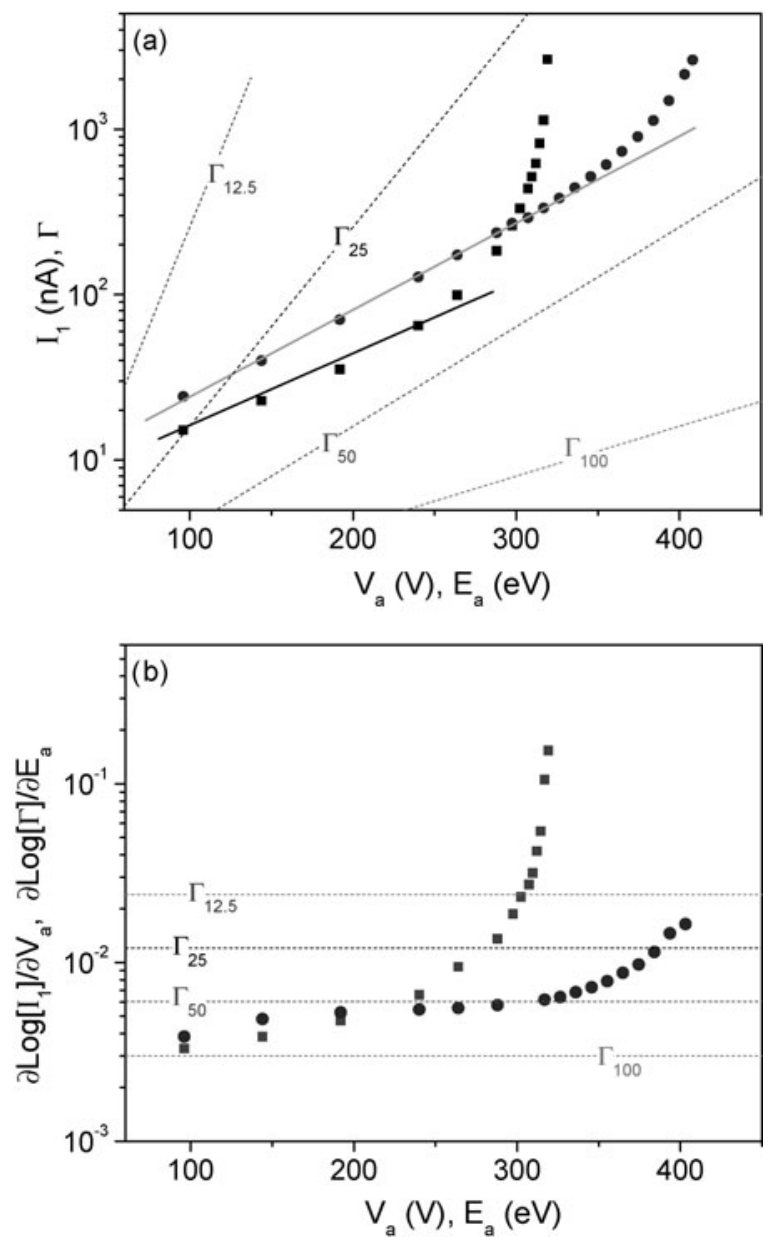

Figure 3. a: Gas cascade output current $\left(I_{1}\right)$ measured as a function of anode bias $\left(V_{a}\right)$ at gas pressures of 24 and $260 \mathrm{~Pa}\left(d_{a}=\right.$ $\left.d_{i}=2.5 \mathrm{~mm}, E_{b}=5 \mathrm{keV}, I_{b}=200 \mathrm{pA}, d_{W}=4 \mathrm{~mm}, B_{m}=253 \mathrm{mT}\right)$. Solid lines indicate the initial slopes of $I_{1}\left(V_{a}\right)$. Dashed lines show the maximum theoretical gain $(\Gamma)$ expected when the sole contribution to the gas cascade is electron ionization of the gas, plotted as a function of total energy $\left(E_{a}\right)$ supplied by the anode potential field when the mean energy cost per electron liberated in the gas cascade $\left(\bar{E}_{i}\right)$ is fixed at $12.5,25,50$, and $100 \mathrm{eV}$. b: Slopes of $\log \left[I_{1}(V)\right]$ and $\log \left[\Gamma\left(E_{a}\right)\right]$ plotted as a function of $V_{a}$ and $E_{a}$, respectively.

peak with a maximum at a pressure of approximately $27 \mathrm{~Pa}$. This low pressure peak (LPP) cannot be accounted for by primary gas ionization processes (Thiel et al., 2006).

Figure 3a shows plots of the gas cascade output current measured as a function of anode bias $\left(V_{a}\right)$ at gas pressures of $24 \mathrm{~Pa}$ (near the LPP) and $260 \mathrm{~Pa}$ (away from the LPP maximum). The results show that gas gain has exponential and super-exponential dependencies on anode bias under conditions low and high bias, respectively (at each pressure, maximum gain was limited by dielectric breakdown of the gas). The initial exponential trends are illustrated by straight line fits to the log-linear plots shown in Figure 3a. The 


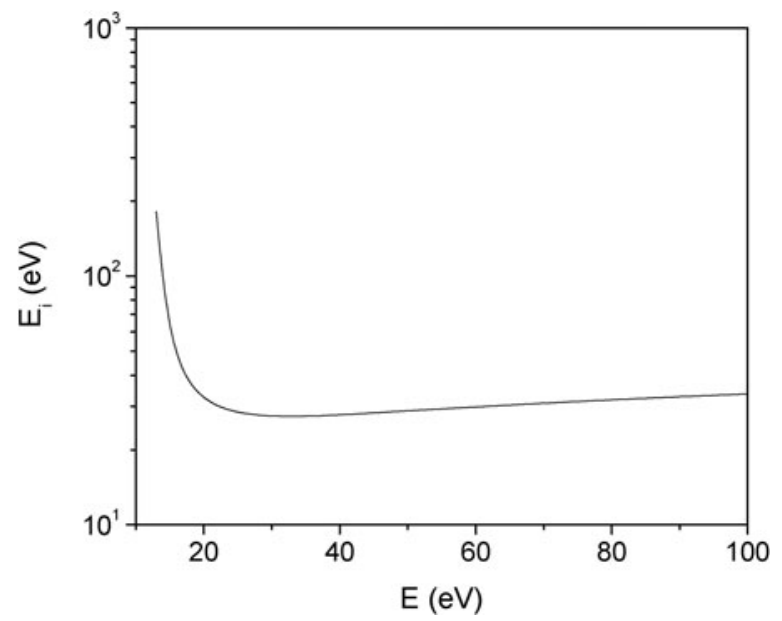

Figure 4. Mean electron energy loss per ionization $\left(\bar{E}_{i}\right)$ calculated as a function of the energy $(E)$ of an electron traversing water vapor.

super-exponential dependencies, seen as deviations of the experimental data from the fits at high biases, cannot be accounted for by primary gas ionization processes. To validate this point, let us consider the maximum theoretical gain $(\Gamma)$ of a primary gas amplification process (i.e., one driven solely by electron ionization of the gas). $\Gamma$ is limited by the total energy $\left(E_{a}=\mathrm{eV}_{a}\right.$, where $e$ is the charge of an electron) supplied to cascading electrons by the electric field created by the anode potential $V_{a}$ (Thiel et al., 1997; Thiel, 2004):

$$
\Gamma=2^{\left(E_{a} / \bar{E}_{i}\right)},
$$

where $\bar{E}_{i}$ is mean energy cost per electron liberated in the gas cascade, assuming that each ionization event generates a single additional free electron in the gas. In the case of impact ionization, $\bar{E}_{i}$ is always greater than the ionization threshold of the gas $\left(12.6 \mathrm{eV}\right.$ in the case of $\mathrm{H}_{2} \mathrm{O}$ ) (Itikawa \& Mason, 2005) due to momentum transfer accompanying ionization events, and competing nonionizing inelastic processes such as vibrational, rotational, and electronic excitations of the gas. $\bar{E}_{i}$ is given by

$$
\bar{E}_{i}=\bar{E}_{e} \frac{\sigma_{e}}{\sigma_{i}}+E_{I}
$$

where $\bar{E}_{e}$ is the cross-section-weighted average of all possible excitation losses, $\sigma_{e}$ and $\sigma_{i}$ are the total excitation and ionization cross sections, respectively, and $E_{I}$ is the average energy loss per ionization collision, including momentum transfer (a review of all major electron- $\mathrm{H}_{2} \mathrm{O}$ collision processes and the corresponding cross sections can be found in Itikawa \& Mason, 2005). Figure 4 shows a plot of $\bar{E}_{i}$ as a

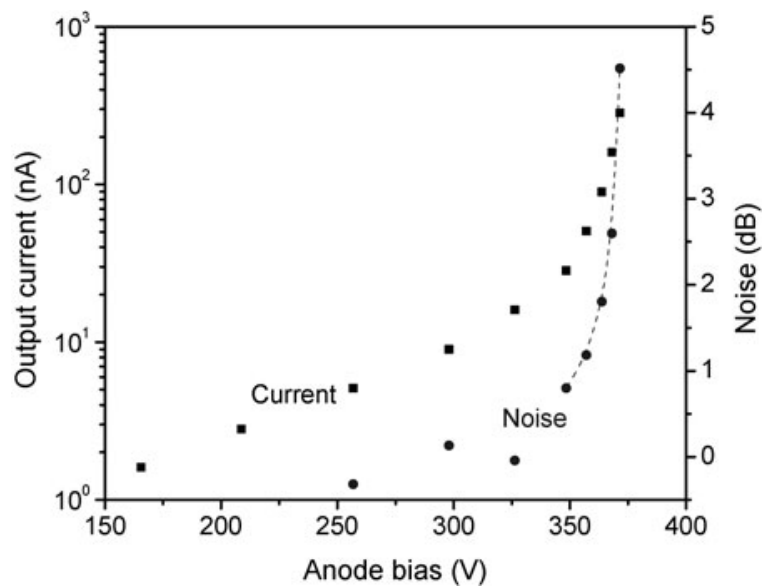

Figure 5. Gas cascade output current and noise (referenced to the mean noise at anode biases smaller than $300 \mathrm{~V}$ ) measured as a function of anode bias. The dashed line highlights a high noise regime at high anode biases $\left(d_{a}=2.5 \mathrm{~mm}, d_{i}=2.0 \mathrm{~mm}, E_{b}=\right.$ $\left.5 \mathrm{keV}, I_{b}=189 \mathrm{pA}, d_{W}=5 \mathrm{~mm}, P=50 \mathrm{~Pa}\right)$.

function of the kinetic energy of the impacting electron. The minimum theoretical value of $\bar{E}_{i}$ is approximately $26 \mathrm{eV}$ for an impact energy of just over $20 \mathrm{eV}$. We note that this energy corresponds to the swarm energy of the primary gas ionization cascade obtained from Monte Carlo simulations of a cascade generated by a constant electric field (Thiel et al., 1997) ("swarm conditions" are characterized by a steady state electron kinetic energy distribution attained when the energy loss rate of electrons traversing the gas is equal to the energy gain from the detector electric field).

Plots of $\Gamma\left(E_{a}\right)$ calculated using equation (1) for $\bar{E}_{i}$ values of $12.6,25,50$, and $100 \mathrm{eV}$, and the corresponding derivatives $\partial \log (\Gamma) / \partial E_{a}$ are shown in Figures $3 \mathrm{a}$ and $3 \mathrm{~b}$, respectively. The experimental data in Figure 3 show that in the exponential amplification regime (fitted by solid lines), $\bar{E}_{i}$ is greater than the theoretical minimum of $\sim 26 \mathrm{eV}$. Conversely, in the super-exponential regime, $\bar{E}_{i}$ decreases below $26 \mathrm{eV}$. Furthermore, at the LPP maximum of $26 \mathrm{~Pa}$ and biases greater than $\sim 300 \mathrm{~V}, \bar{E}_{i}$ is smaller than $12.5 \mathrm{eV}$, the ionization threshold of $\mathrm{H}_{2} \mathrm{O}$. Hence, the total gas amplification cannot be explained solely by a primary ionization cascade. Super-exponential gain behavior, however, is characteristic of feedback-assisted amplification and secondary ionization processes. In the gas cascade, feedback can arise when an excited or ionized gas molecule (whose populations scale with the primary ionization gain) migrates to a region of reduced potential and injects a free electron into the gas upon de-excitation. That electron initiates a new cascade which in turn creates excited and ionized species. However, the initial coordinates of the free electron in the field are not deterministic, and the associated gain is expected to have a stochastic component that should generate additional noise at the amplifier output. Figure 5 shows 
plots of the gas cascade output current and noise measured as a function of anode bias using the methodology described in Tileli et al. (2009). The data clearly show that the super-exponential increase in gain observed at high anode biases correlates with an even faster increase in noise, consistent with the suggestion of a feedback path in the gas amplification process.

In general, secondary ionization can take place through multiple, parallel pathways, including Auger and photoelectron emission processes accompanying the neutralization of gaseous ions at or near solid surfaces (Hagstrum, 1978; Hahn, 1997), and other processes that accompany the relaxation of metastable excited species (von Engel, 1955). The fact that an LPP is observed only in magnetic field assisted gaseous secondary electron detectors provides some clues as to its origin. Compared to free electrons, positive gaseous ions are affected weakly by the magnetic field used in MIL ESEM. Regardless of pressure, the ions drift to and neutralize at solid surfaces, as in magnetic field-free gas cascade detectors, and are therefore unlikely to be responsible for the observed feedback. However, free electrons can also be generated by metastable species excited in the gas cascade. In order for this to be possible, a sufficiently long-lived excited species with a potential energy in excess of the ionization threshold of the gas must exist. In that case, a free electron can be generated during an autoionization event triggered by a collision between the metastable species and another gas molecule. At $26 \mathrm{~Pa}$, the maximum of the LPP kinetic gas theory predicts a molecular collision meanfree-path of approximately $0.5 \mathrm{~mm}$. In the case of the compact magnetic field-assisted gas cascade detector used here and described previously in Thiel et al. (2006), this distance is sufficient for excited species created in the densest part of the cascade (i.e., near the anode) to de-excite in a region of significantly reduced electric potential, enabling electrons thus generated to contribute to feedback in the gas cascade. At pressures beyond the LPP, feedback is reduced because the mean-free-path decreases exponentially with pressure (falling to around $60 \mu \mathrm{m}$ at $240 \mathrm{~Pa}$ ). Conversely, at pressures below the LPP maximum, feedback is insufficient to make up for the decrease in total amplification with decreasing pressure (Thiel et al., 2006). Hence, metastable de-excitation may account for the observed feedback, but further studies are clearly needed to evaluate all the possible secondary ionization mechanisms and elucidate their relative contributions to feedback in the gas cascade.

The existence of anomalous gain and noise behavior suggests that detector geometries and operating conditions can be used to optimize the signal and noise characteristics of MIL ESEM systems. This is expected to be beneficial in demanding imaging applications requiring the use of low electron doses, such as critical dimension metrology (Postek et al., 2004; Postek \& Vladar, 2004), imaging of beam sensitive materials (e.g., polymers and biological tissue), and real-time imaging of nanostructure growth by gas mediated electron beam induced deposition (Toth et al., 2007).

\section{CONCLUSION}

The gas cascade amplification process used in magnetic immersion lens ESEM exhibits anomalous gain behavior that cannot be accounted for by primary gas ionization processes. The underlying mechanism is attributed to a feedback path in the gas cascade that dominates gain under the conditions typically used for ultra-high-resolution ESEM and gives rise to novel dependencies of the imaging signal and noise on microscope operating parameters. Feedback is ascribed tentatively to the generation of free electrons upon de-excitation of metastable species in the gas cascade.

\section{REFERENCES}

Baker, F.S., Craven, J.P. \& Donald, A.M. (2003). The environmental scanning electron microscope and its applications. In Techniques for Polymer Organisation and Morphology Characterisation, Pethrick, R.A. \& Viney, C. (Eds.), pp. 111-139. West Sussex, UK: John Wiley \& Sons.

Bogner, A., Guimaraes, A., Guimaraes, R.C.O., Santos, A.M., Thollet, G., Jouneau, P.H. \& Gauthier, C. (2008). Grafting characterization of natural rubber latex particles: Wet-STEM imaging contributions. Colloid Polymer Sci 286, 1049-1059.

Folch, A., Servat, J., Esteve, J., Tejada, J. \& Seco, M. (1996). High-vacuum versus "environmental" electron beam deposition. J Vac Sci Technol B 14, 2609-2614.

Folch, A., Tejada, J., Peters, C.H. \& Wrighton, M.S. (1995). Electron-beam deposition of gold nanostructures in a reactive environment. Appl Phys Lett 66, 2080-2082.

Hagstrum, H.D. (1978). Studies of adsorbate electronic structure using ion neutralization and photoemission spectroscopies. In Electron and Ion Spectroscopy of Solids, Fiermans, L., Vennik, J. \& Dekeyser, W. (Eds.), pp. 273-323. New York: Plenum Press.

HaHn, Y. (1997). Electron-ion recombination processes-An overview. Rep Prog Phys 60, 691-759.

Itikawa, Y. \& Mason, N. (2005). Cross sections for electron collisions with water molecules. J Phys Chem Ref Data 34, 1-22.

Kucheyev, S.O., Toth, M., Baumann, T.F., Hamza, A.V., Ilavsky, J., Knowles, W.R., SaW, C.K., Thiel, B.L., Tileli, V., vaN BuUren, T., Wang, Y.M. \& Willey, T.M. (2007). Structure of low-density nanoporous dielectrics revealed by low-vacuum electron microscopy and small-angle X-ray scattering. Langmuir 23, 353-356.

Molhave, K., Madsen, D.N., Dohn, S. \& Boggild, P. (2004). Constructing, connecting and soldering nanostructures by environmental electron beam deposition. Nanotechnology 15, 1047-1053.

Molhave, K., Madsen, D.N., Rasmussen, A.M., Carlsson, A., Appel, C.C., Brorson, M., Jacobsen, C.J.H. \& Boggild, P. (2003). Solid gold nanostructures fabricated by electron beam deposition. Nano Lett 3, 1499-1503.

Noscinovsky, M. \& Bhushan, B. (2008). Patterned nonadhesive surfaces: Superhydrophobicity and wetting regime transitions. Langmuir 24, 1525-1533.

Postek, M.T. \& Vladar, A.E. (2004). New application of variablepressure/environmental microscopy to semiconductor inspection and metrology. Scanning 26, 11-17. 
Postek, M.T., Vladar, A.E., Bennett, M.H., Rice, T. \& Knowles, R. (2004). Photomask dimensional metrology in the scanning electron microscope, part II: High-pressure/environmental scanning electron microscope. J Microlithog Microfab Microsyst 3, 224-231.

Rossi, M.P., Ye, H.H., Gogotsi, Y., Babu, S., Ndungu, P. \& Bradley, J.C. (2004). Environmental scanning electron microscopy study of water in carbon nanopipes. Nano Lett 4, 989-993.

Stelmashenko, N.A., Craven, J.P., Donald, A.M., Terentjev, E.M. \& ThieL, B.L. (2001). Topographic contrast of partially wetting water droplets in environmental scanning electron microscopy. J Microsc-Oxf 204, 172-183.

Stokes, D.J., Thiel, B.L. \& Donald, A.M. (1998). Direct observation of water-oil emulsion systems in the liquid state by environmental scanning electron microscopy. Langmuir 14, 4402-4408.

Thiel, B.L. (2004). Master curves for gas amplification in low vacuum and environmental scanning electron microscopy. Ultramicroscopy 99, 35-47.

Thiel, B.L., Bache, I.C., Fletcher, A.L., Meredith, P. \& DonALD, A.M. (1997). An improved model for gaseous amplification in the environmental SEM. J Microsc-Oxf 187, 143-157.
Thiel, B.L. \& Tотн, M. (2005). Secondary electron contrast in low-vacuum/environmental scanning electron microscopy of dielectrics. J Appl Phys 97, 051101.

Thiel, B.L., Toth, M., Schroemges, R.P.M., Scholtz, J.J., van Veen, G. \& KNowles, W.R. (2006). Two-stage gas amplifier for ultrahigh resolution low vacuum scanning electron microscopy. Rev Sci Instrum 77, 033705.

Tileli, V., Knowles, W.R., Toth, M. \& Thiel, B.L. (2009). Noise characteristics of the gas ionization cascade used in low vacuum scanning electron microscopy. J Appl Phys 106, 014904.

Toтн, M., Knowles, W.R. \& Thiel, B.L. (2006). Secondary electron imaging of nonconductors with nanometer resolution. Appl Phys Lett 88, 023105.

Toth, M., Lobo, C.J., Knowles, W.R., Phillips, M.R., Postek, M.T. \& VladAR, A.E. (2007). Nanostructure fabrication by ultra-high-resolution environmental scanning electron microscopy. Nano Lett 7, 525-530.

von Engel, A. (1955). Ionized Gases. Oxford, UK: Clarendon Press. 